Military Technical College

Kobry El-Kobbah,

Cairo, Egypt

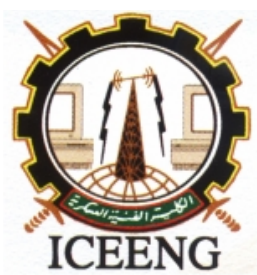

ICEENG

\author{
$8^{\text {th }}$ International Conference \\ on Electrical Engineering
}

ICEENG 2012

\title{
QUALITY ASSESMENT OF WAVELET BASED IMAGE FUSION TECHNIQUES
}

\author{
A. M. El Ejaily ${ }^{*}$, F. El Tohamy**, M.Y. El Nahass ${ }^{* * *}$,
}

Abstract - image fusion is a process of producing a single fused image from a set of input images. The fused image has enhanced information more than any of the input images

In this paper a wavelet based image fusion experiments are carried out, and the impacts of changing various wavelet parameters on the quality of the fused image are investigated.

The fusion of wavelet coefficients is done according to three algorithms. Fusion experiments are performed on QuickBird panchromatic (PAN) and multispectral (MS) images. In order to evaluate the quality of the fused images four quantitive measures of correlation coefficients, root mean square error, information entropy, and standard deviation are used. Fusion methods like IHS transform and brovey transform method are investigated, and their results are compared with that of our experiments based on the using of wavelet transform.

Keywords: image fusion; discrete wavelet transform; decomposition level; quality assessment.

\section{Introduction}

Image fusion is a technique of combining complementary information from multiple images originated from different sources into a single image. The fusion process improves the quality of the fused image compared to the original input images. Image fusion has been used in many fields such as machine vision, medical diagnosis, military applications, and remote sensing applications.

In satellite remote sensing, the sensors provide us with two main types of images: panchromatic image of high spatial resolution and low spectral resolution and multispectral image of high spectral resolution and low spatial resolution. Image fusion can be employed as an effective and inexpensive tool to merge those two main types producing a fused image of high spatial and spectral resolution [1]

. There are many image data fusion methods[2],[3], including intensity-hue- saturation(IHS) transform, principle component analysis (PCA), Brovey transform (BT), two-dimensions discrete wavelet transform(2D-DWT), etc.

Recent researches showed that the wavelet based image fusion techniques outperform the standard image fusion methods[4],[5] . 
In this paper the main objective is to apply 2D-DWT method in image fusion and study the effects of changing some of the wavelet transform parameters on the quality of the fused image. The fusion is also performed using HIS d BT methods, and the obtained results are compared with that of wavelet-based technique.

*Ph.D. student Libyan armed forces.

${ }^{* *}$ Ph.D. Egyptian armed forces.

${ }^{* * \star}$ Prof. Alazhar University, Cairo, Egypt

\section{Theory of the two-dimensional discrete wavelet transform.}

The wavelet decomposition of two-dimensional image commonly used discrete wavelet transform. According to Mallat fast algorithm [6], a low pass filter $(\mathrm{H})$ and a high pass filter(G) are used to decompose the original two-dimensional image $(A)$ on scale $\mathrm{j}$-1into four sub-images as follows

$$
\begin{aligned}
& A_{j}=H_{m} H_{n} A_{j-1} \\
& D_{j}^{H}=G_{m} H_{n} A_{j-1} \\
& D_{j}^{V}=H_{m} G_{n} A_{j-1} \\
& D_{j}^{D}=G_{m} G_{n} A_{j-1}
\end{aligned}
$$

Where

$A_{j}, D_{j}^{H}, D_{j}^{V}$ and $D_{j}^{D}$ are the low-frequency components, vertical, horizontal, diagonal direction high frequency components respectively.

Correspondingly, the wavelet reconstruction formula is as follows:

$$
C_{j-1}=H_{m}^{*} H_{n}^{*} C_{j}+H_{m}^{*} G_{n}^{*} D_{j}^{H}+G_{m}^{*} H_{n}^{*} D_{j}^{V}+G_{m}^{*} G_{n}^{*} D_{j}^{D}
$$

Where

$C$ is the reconstructed two-dimensional image

$H^{*}, G^{*}$ are the conjugate transposed matrix of $H, G$.

Wavelet transform divides the original image into approximate and detail components through different filters, namely, low-frequency part and high-frequency part. High-frequency components are divided into horizontal, vertical and diagonal three components. Further decomposition of the image is done only for the low-frequency component and, if the image is decomposed using $\mathrm{N}$-layer wavelet decomposition, there will be $(3 \mathrm{~N}+1)$ different frequency components, including the $3 \mathrm{~N}$ high frequency components and a low frequency component. Fig. 1 shows 3 level of 2D-DWT. 


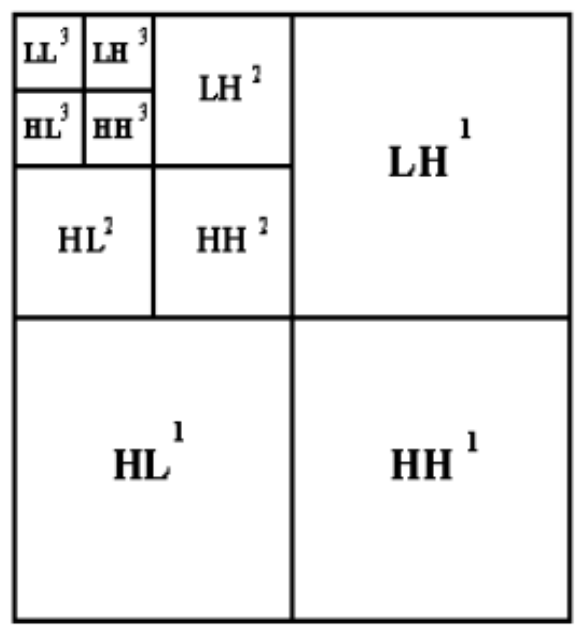

Figure 1 2D-DWT with three levels of decompositions

As the decomposition levels increase, the frequency range is gradually reduced, and the calculation amount of image fusion is also greatly increased, so 1-5 levels of wavelet decomposition were chosen to study in the experiment.

\section{Wavelet Based Image Fusion}

Fusion methods based on 2D-DWT are done by combine decomposition coefficients of two or more source images using a certain fusion rule then a backward transform is performed on the combined coefficients resulting in the fused image. A general scheme for 2D-DWT based fusion methods is shown in Fig.2. In this paper three experiments are carried out to study the effects of changing of various wavelet parameters on the quality of the fused image.

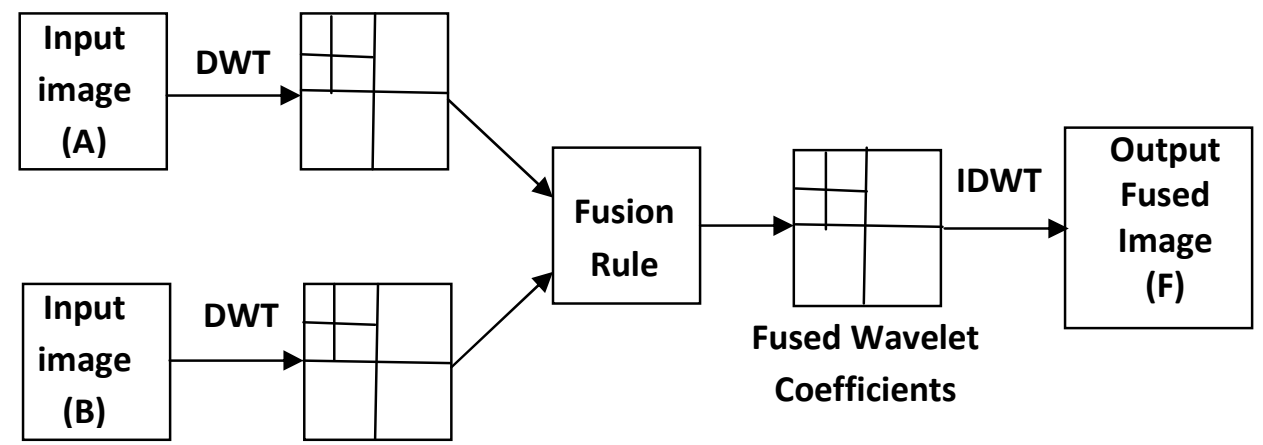

Figure 2: general scheme of wavelet based image fusion

\section{A. Study the effect of fusion rule}


In this experiment the input images to be fused named A \& B, are transformed using the same wavelet base $(\mathrm{db} 2)$, and transformed to the same number of levels. Let The transformed coefficient are fused according to three fusion rules then the fused image $\mathrm{F}$ is constructed through the inverse transform.

For the all of the fusion rules, the approximation coefficients of the fused image are constructed by averaging out the approximation coefficient of the sources images A \& B, the details coefficients are calculated as follows:

Fusion rule 1: Since large absolute values of transform coefficients corresponds to sharper brightness changes [7], the details coefficients are constructed by comparing the details of the source images and we select that has maximum absolute value [8].

Fusion rule 2: the details coefficients of fused details coefficient are constructed based on maximum gradient of that of the sources images [9].

The average gradient magnitude $(\bar{G})$ is defined as:

$$
\bar{G}=\left[\left(\frac{\partial f(i, j)}{\partial x}\right)^{2}+\left(\frac{\partial f(i, j)}{\partial y}\right)^{2}\right]^{1 / 2}
$$

Where $\partial f(i, j) / \partial x$ and $\partial f(i, j) / \partial y$ are one order differential of corresponding wavelet coefficients in $\mathrm{x}$ and $\mathrm{y}$ direction respectively.

The fused coefficients are obtained by setting each coefficient equal to the corresponding input image wavelet coefficient that has the greatest average gradient.

Fusion rule 3: in this rule we replace all of the MS image with that of the panchromatic image [10], so we select all the panchromatic details as a details coefficients of the fused image.

\section{B. Study the effect of the number of levels of decomposition:}

In this experiment the input images A \& B are transformed using the same wavelet base $(\mathrm{db} 2)$, the of decompositions increases from just single level up to five level of decomposition. Fusion rule 3 is used to synthesize the coefficient of the fused image at all levels of decomposition.

\section{Study the effect of the selection of wavelet base:}

The effect of changing the wavelet base is studied by transforming the input images using different wavelet basis ( $\mathrm{db} 2, \mathrm{db} 5, \mathrm{db} 1, \mathrm{sym} 2$, bior1.1 and coif2) with three levels of decomposition and then Fusion rule 3 is used to synthesize the coefficients of the fused image.

\section{Quality evaluation of the fused images}

For assessing the quality of the obtained fused images we measure the information entropy (EN) and standard deviation (STD) of each band of the fused image and the gray scale version of the fused image (GF). Correlation coefficients (CC) between fused image and PAN image, and 
between the PAN image and gray scale version of the fused image are calculated. Finally we calculate the root mean square error (RMSE) between fused image and original MS image, and between the PAN image and gray scale version of the fused image.

The information entropy (EN) can be calculated according to the following equation [11]:

$$
E N=-\sum_{g=0}^{L-1} p(g) \log _{2} p(g),
$$

where $\mathrm{p}(\mathrm{g})$ is the probability of gray level $\mathrm{g}$, and the range of $\mathrm{g}$ is $[0, \ldots, \mathrm{L}-1]$. And $\mathrm{L}$ is the maximum value of the gray levels.

The standard deviation of the image $\mathrm{A}$ is one of the important measures, and it can be calculated according to the following equation [11]:

$$
S T D=\left(\frac{1}{n-1} \sum_{i=1}^{n}\left(A_{i}-\bar{A}\right)^{2}\right)^{\frac{1}{2}}
$$

where $\bar{A}$ denotes the mean of image pixels, $\mathrm{A}_{\mathrm{i}}$ denotes the pixel of image, and $\mathrm{n}$ is the total number of the image pixels. Generally large value of the STD denotes that information content of the image are high, and more information are gained.

The correlation coefficients between two images measure the closeness or similarity between two images. It can vary between $[-1: 1]$. A value close to +1 indicates that the two images are very similar, while a value close to -1 indicates that they are highly dissimilar. The formula to compute the correlation between two images $\mathbf{A} \& \mathbf{B}$ is given by [11]:

$$
\operatorname{Corr}(A / B)=\frac{\sum_{i=1}^{N} \sum_{j=1}^{N}\left(A_{i, j}-\bar{A}\right)\left(B_{i, j}-\bar{B}\right)}{\sqrt{\sum_{i=1}^{N} \sum_{j=1}^{N}\left(A_{i, j}-\bar{A}\right) \sum_{i=1}^{N} \sum_{j=1}^{N}\left(B_{i, j}-\bar{B}\right)}},
$$

The root mean square error measures the difference between two images, small amount of RMSE means the images are closed to each other. The formula for computing RMSE between two images A \& B is given by[11]:

$$
R M S E=\sqrt{\frac{1}{M N} \sum_{i=1}^{N} \sum_{j=1}^{M}|A(i, j)-B(i, j)|^{2}}
$$


Where $(i, j)$ denotes a given pixel, and NxM is the size of the image.

\section{Experimental results and discussion}

Two subsets of 400x400 pixels of the QuickBird PAN and MS images of a city Musrate in North West of Libya are used to conducts the image fusion experiments explained in section III and shown in Fig.3.

Fig.4 shows the obtained fused images corresponding to the use of different levels of wavelet decomposition in the transformation of the input images. The resultant fused image of using different fusion rules explained in section III are shown in Fig.5. Fig.6 shows the obtained fused images when we change the wavelet function. Fig.7 shows the fused images of IHS transform method, brovey transform method and that of wavelet-based method.

Table1 presents the results of the objective evaluation of the fused images resulted from different levels of wavelet decomposition, and the result of the objective evaluation of fused images produced by using different fusion rules are given in table.2. Table. 3 presents the results of the objective evaluation of the fused images resultant from the use of different wavelet base functions. Table. 4 gives the results that compare wavelet-based method with HIS, PCA and brovey transform methods.

It is clear from table. 1 that the quality of the fused image is improved as we increase the level of decomposition at the expense of calculation complexity, and the results of table. 2 shows the better quality is achieved when we use fusion rule 3 in synthesizing the wavelet coefficients of the fused image. Since the results of table. 3 are much closed to each other, we can say that no matter what kinds of wavelet function are used. Finally the results of table.4 show that the wavelet-based image fusion method has a better performance than IHS and brovey transform methods.

\section{Conclusion}

In this paper three wavelet-based experiments of merging a remotely sensed digital satellite images are conducted. The objective of those experiments is to study the effect of changing various wavelet transform parameters on the quality of the fused image. We conclude that the fused image quality is improved as we increase the number of the levels of wavelet decompositions while the computer calculations are significant increase. More over the using of fusion rule in which all of details of the MS image are replaced with that of PAN image gives better result than the other tested fusion rules and we can also conclude that no matter what kinds of wavelet function are used to perform the experiments. Finally the use of wavelet-based image fusion method of proper choices of wavelet parameters give better result than standard image fusion methods like IHS and brovey transform methods. 


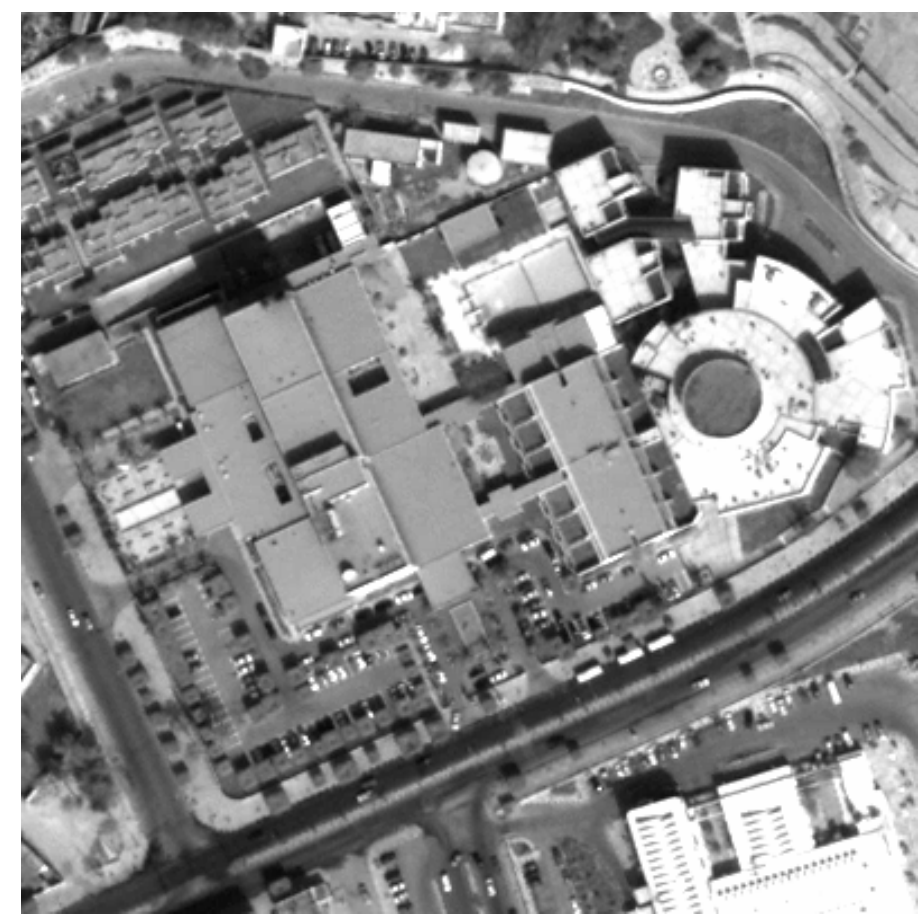

(a) 


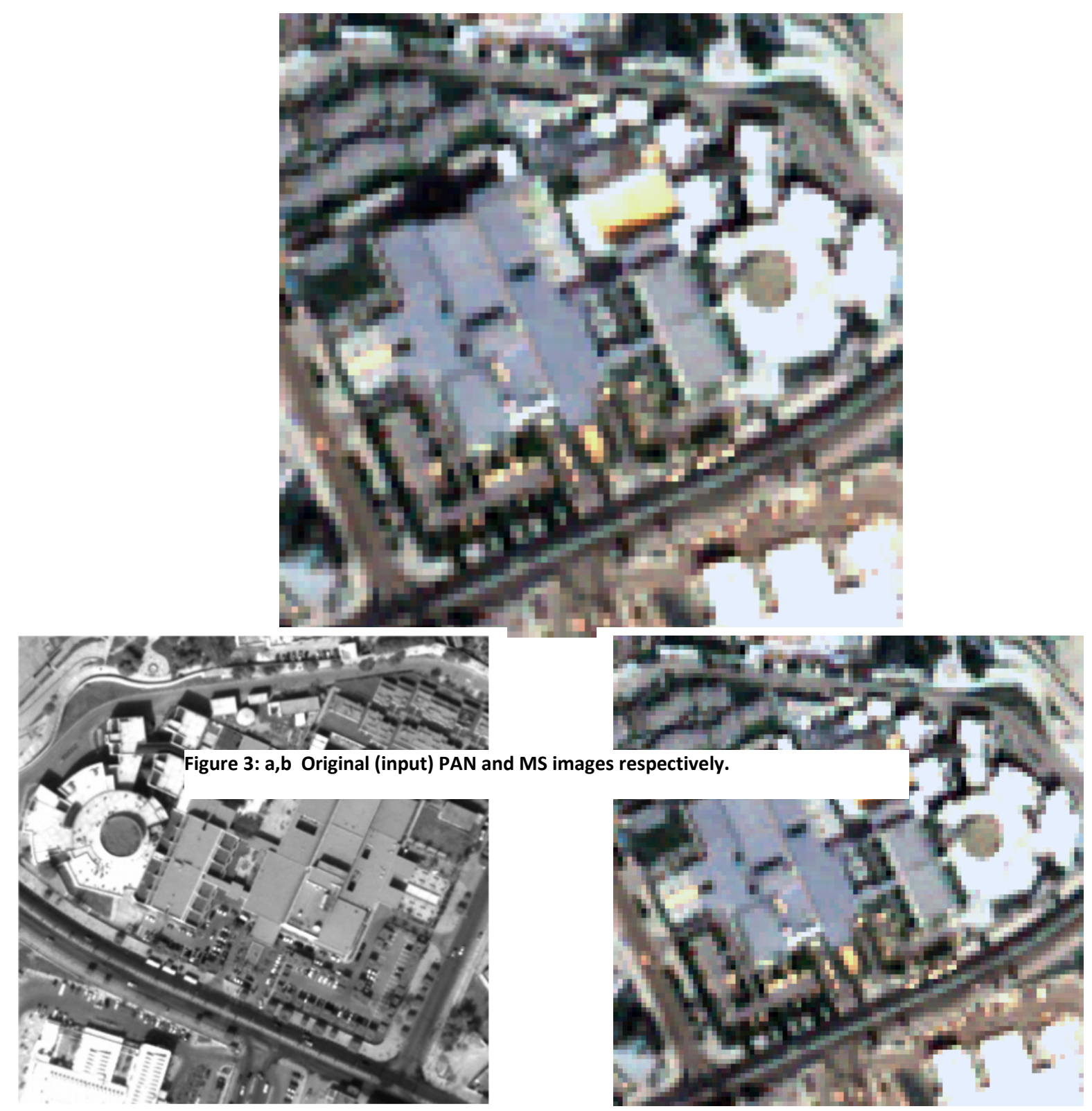

(a)

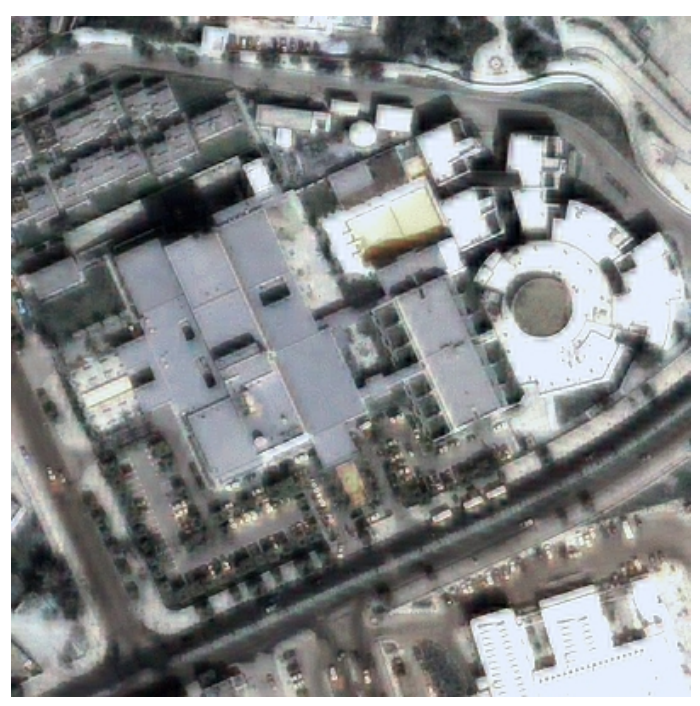

(b)

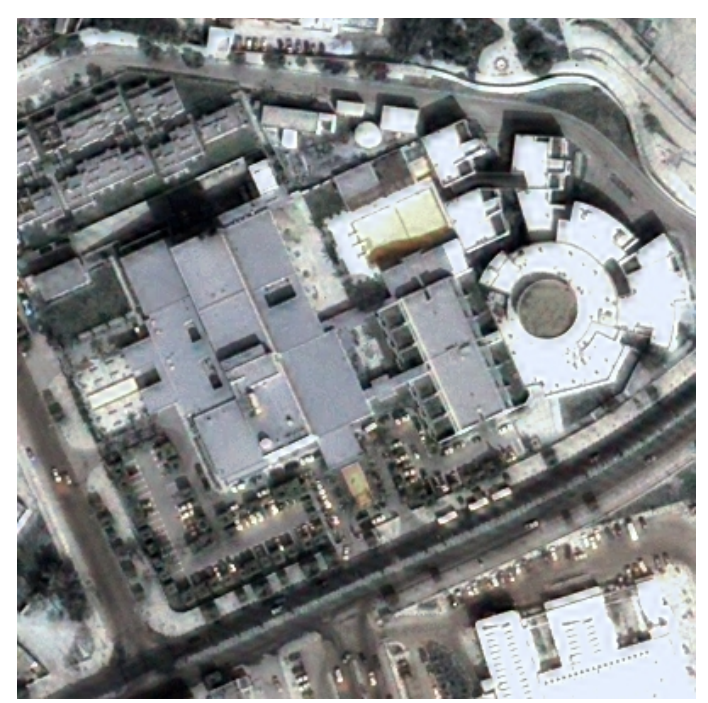


(c)

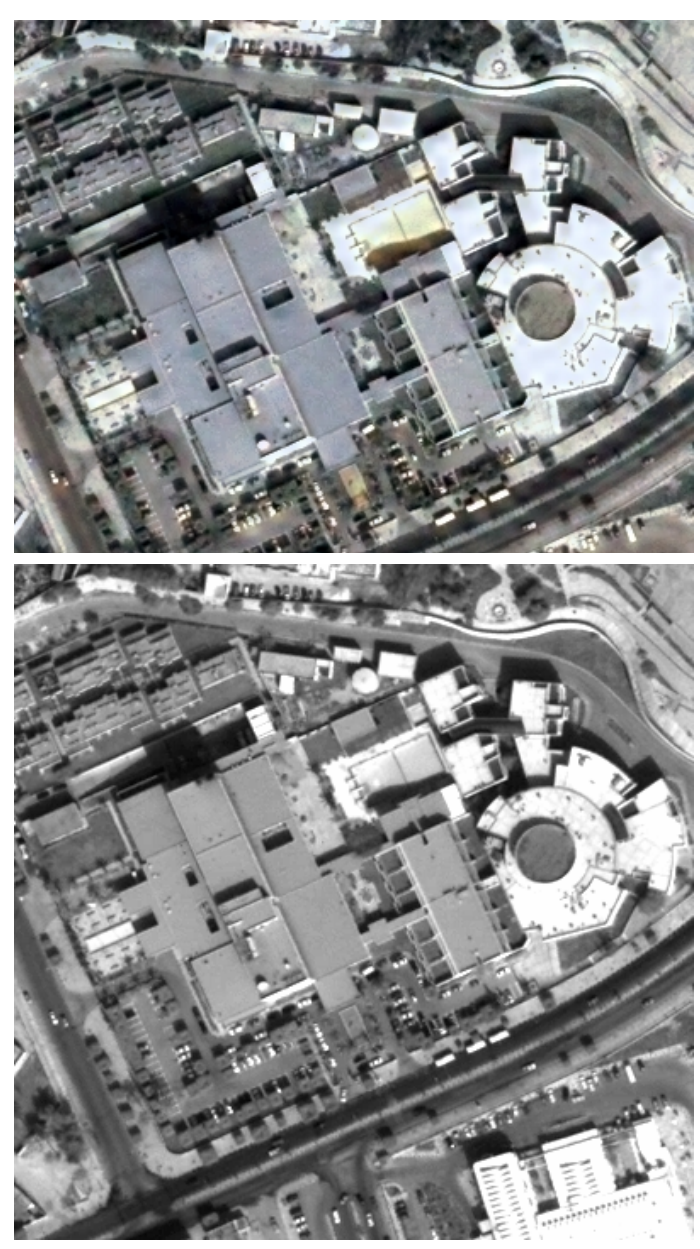

(a)

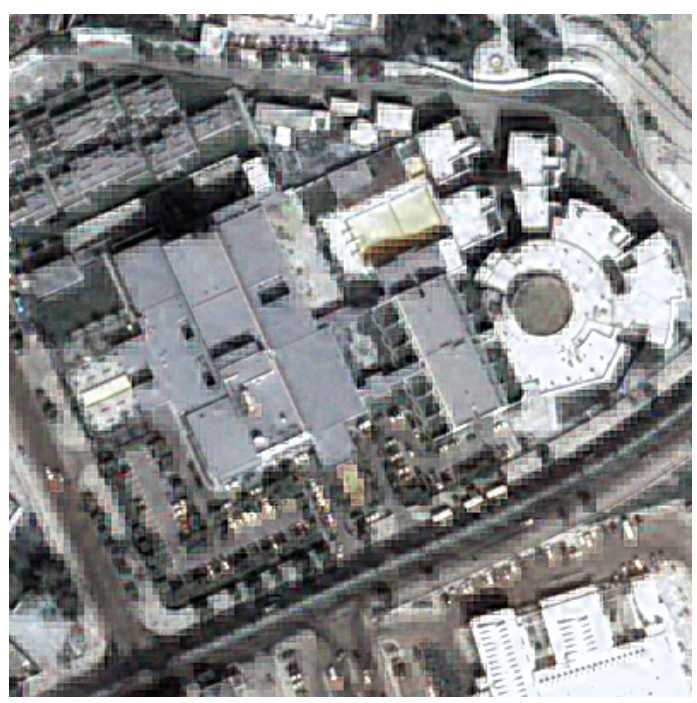

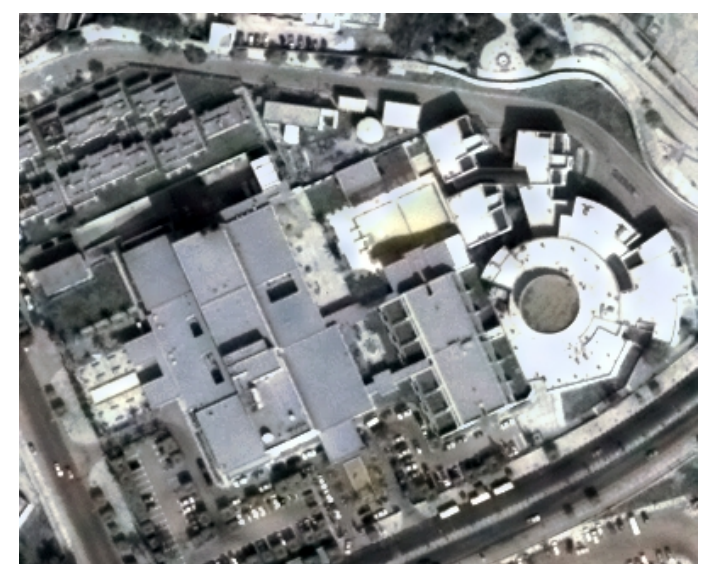

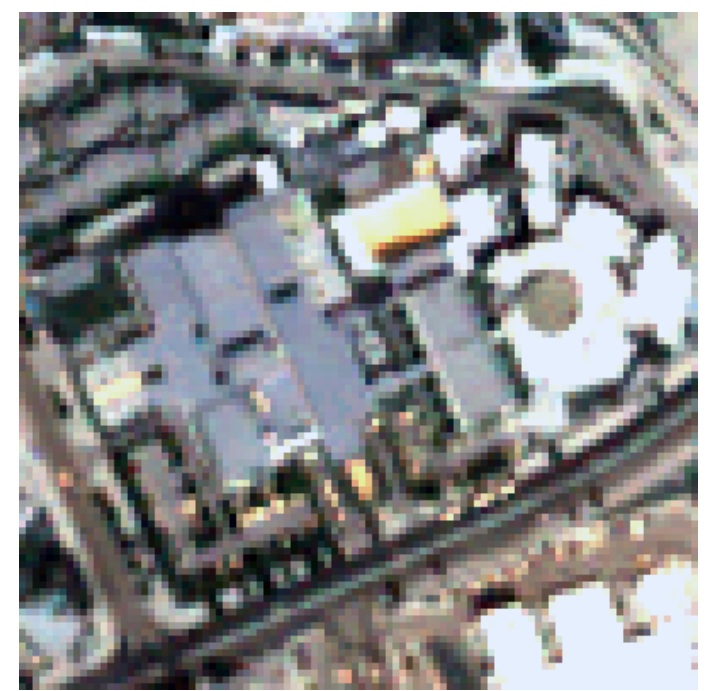

(b)

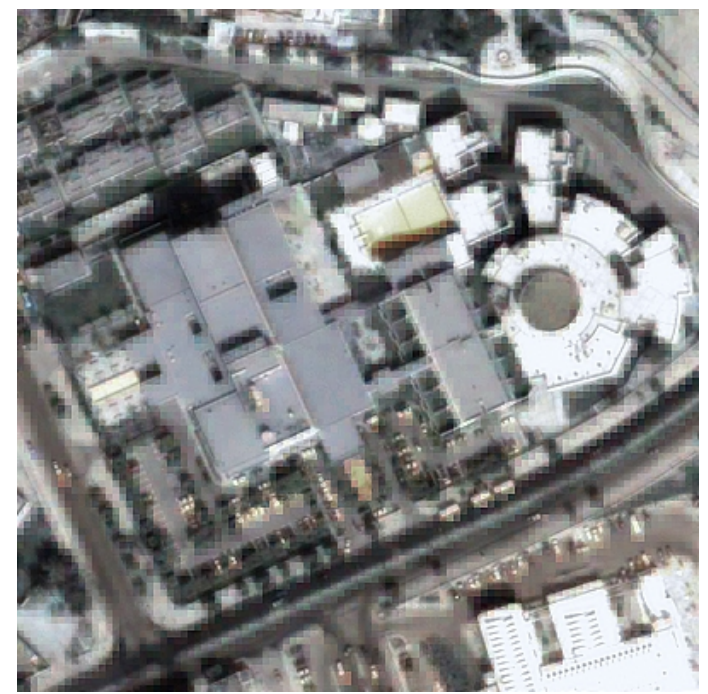

(d) 
(c)

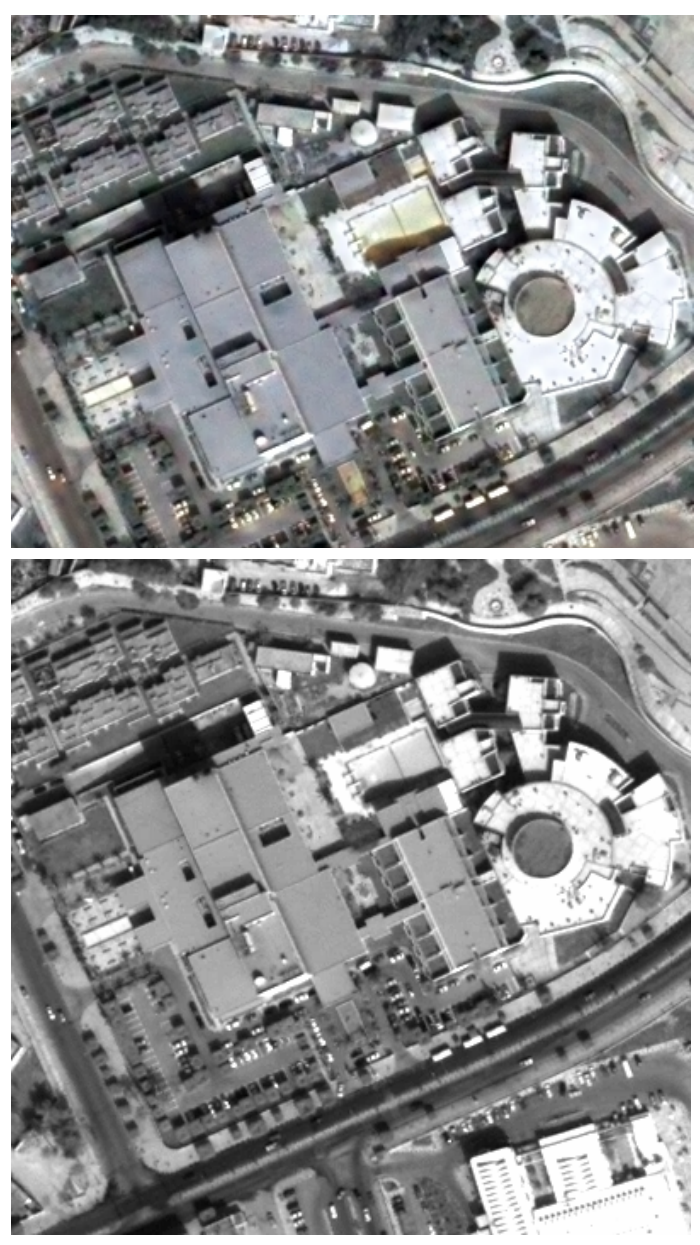

(a)

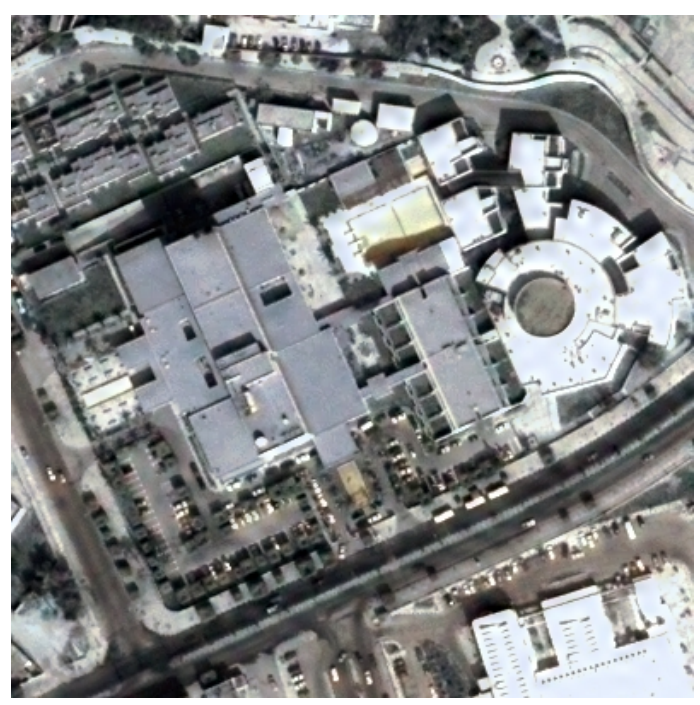

$\checkmark$ and MS

n absoluti Is coefficie

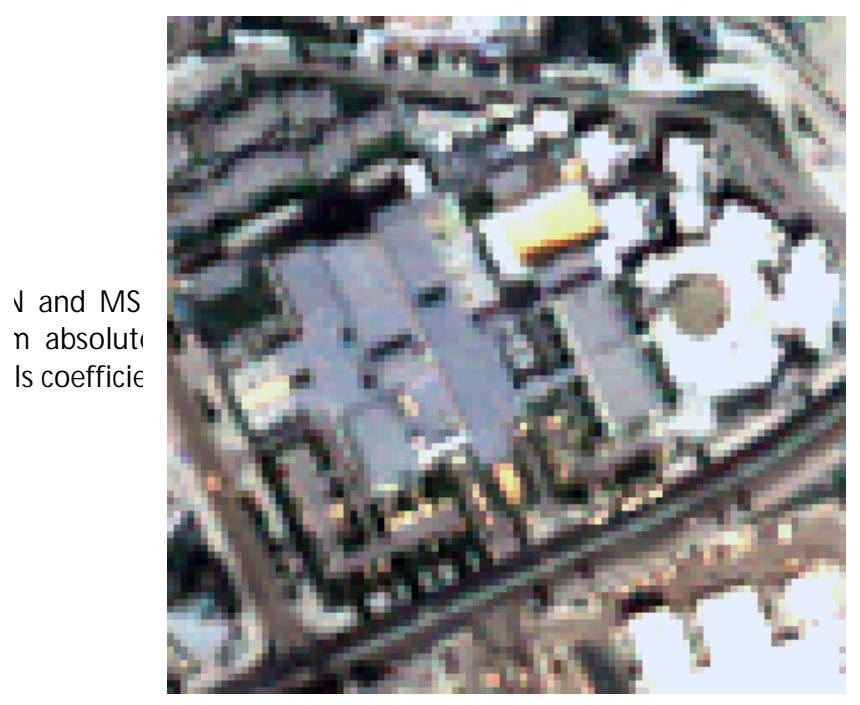

(b)

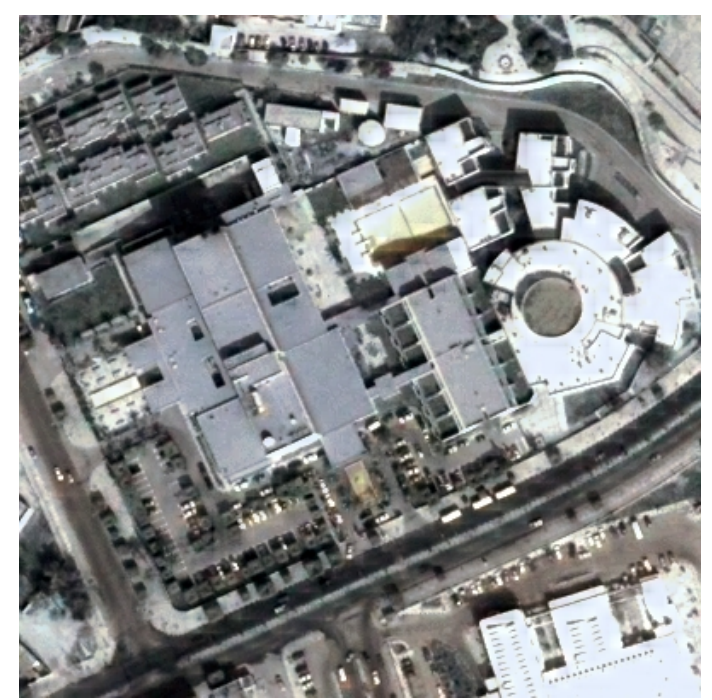

(d) 
(c)

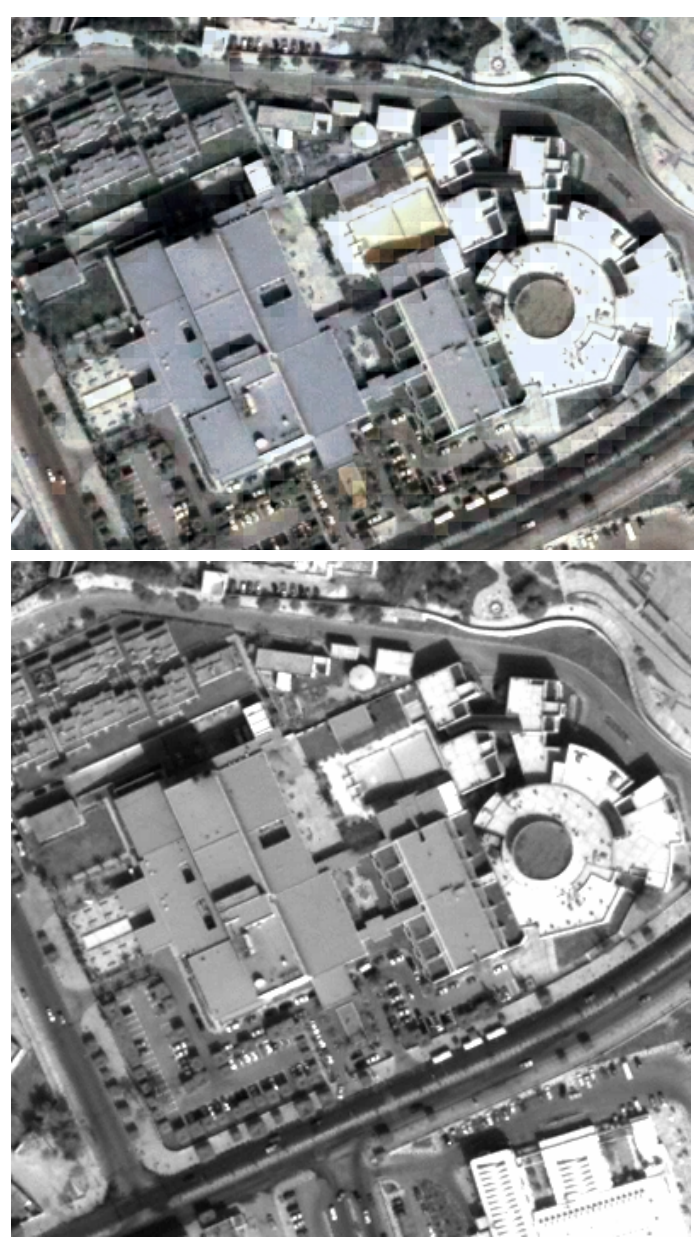

(a)

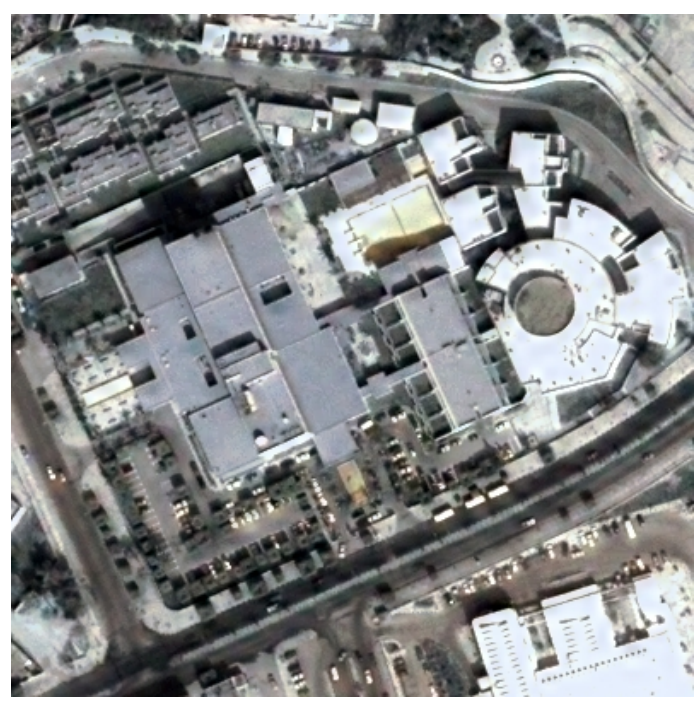

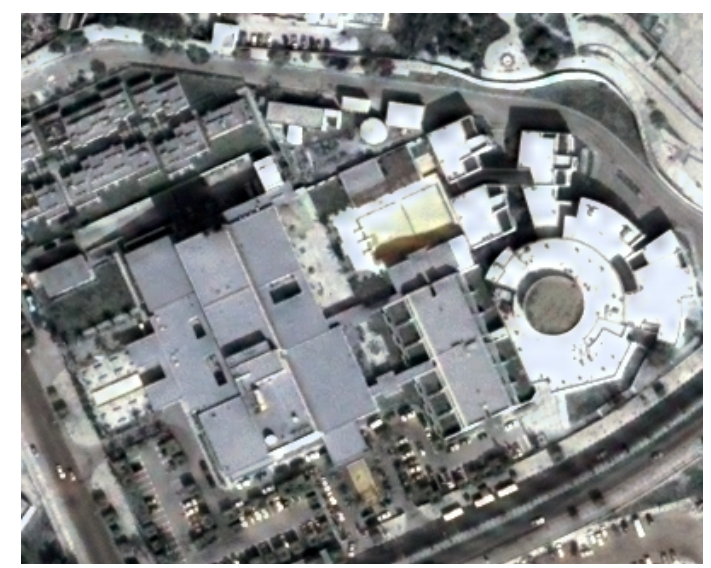

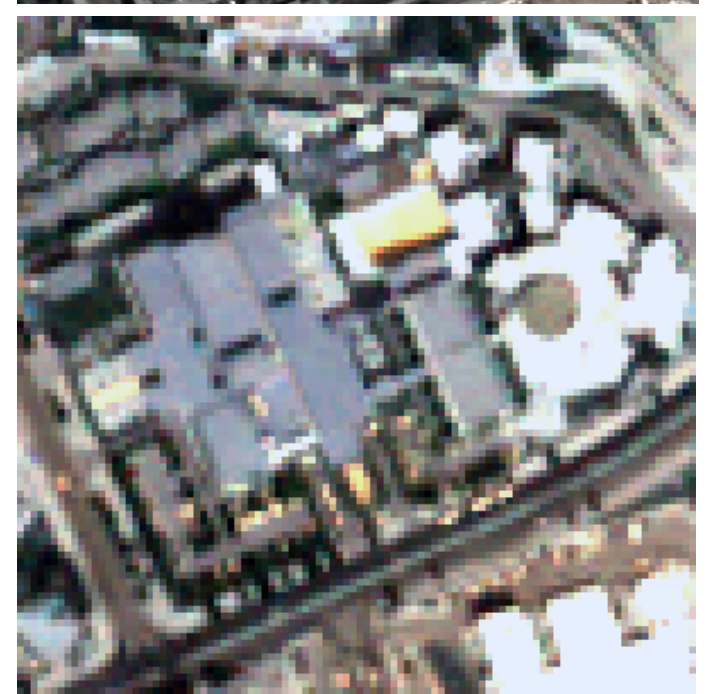

(b)

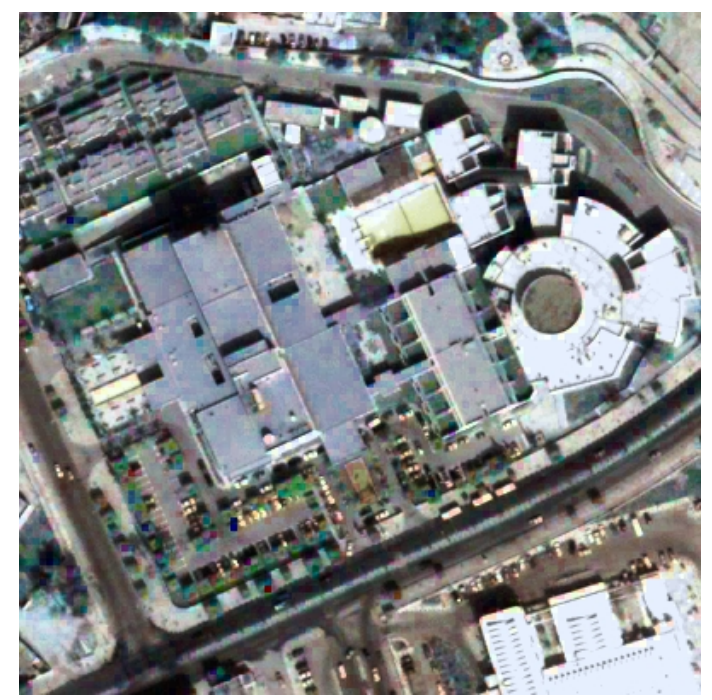

(d) 
(c)

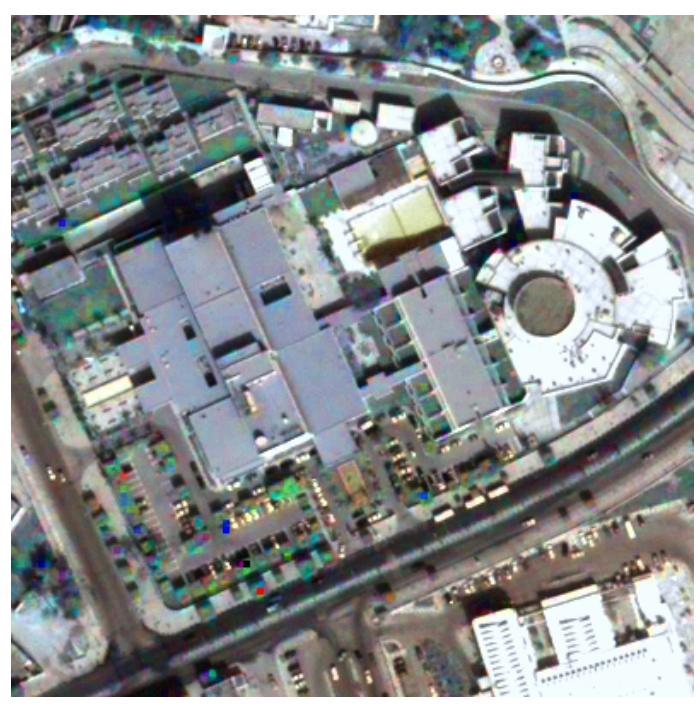

(e)

Figure 7: a,b Original (input) PAN and MS images respectively. c,d,e Fused images using DWT, HIS, and brovey transform method respectively. 
Table 1: statistical indicators of CC, EN, RMSE, STD for fused images resulted from changing level of Decompositions

\begin{tabular}{|c|c|c|c|c|c|c|}
\hline & & One level & $\begin{array}{c}\text { Two } \\
\text { levels }\end{array}$ & $\begin{array}{l}\text { Three } \\
\text { levels }\end{array}$ & $\begin{array}{c}\text { Four } \\
\text { levels }\end{array}$ & $\begin{array}{c}\text { Five } \\
\text { levels }\end{array}$ \\
\hline \multirow{4}{*}{$\mathrm{CC}$} & $\left(\mathbf{F}_{1} \mid \mathbf{P A N}\right)$ & 0.9878 & 0.9904 & 0.9931 & 0.9961 & 0.9977 \\
\hline & $\left(\mathrm{F}_{2} \mid \mathbf{P A N}\right)$ & 0.9905 & 0.9930 & 0.9954 & 0.9976 & 0.9988 \\
\hline & $\left(\mathbf{F}_{3} \mid \mathbf{P A N}\right)$ & 0.9911 & 0.9936 & 0.9958 & 0.9979 & 0.9969 \\
\hline & (GF|PAN) & 0.9902 & 0.9920 & 0.9937 & 0.9957 & 0.9969 \\
\hline \multirow{4}{*}{ Entropy } & $\left(\mathbf{F}_{1}\right)$ & 7.77074 & 7.8011 & 7.8426 & 7.8408 & 7.8254 \\
\hline & $\left(\mathbf{F}_{2}\right)$ & 7.7457 & 7.8249 & 7.8588 & 7.8582 & 7.8172 \\
\hline & $\left(\mathbf{F}_{3}\right)$ & 7.7123 & 7.7300 & 7.7252 & 7.7063 & 7.6410 \\
\hline & (GF) & 7.7483 & 7.8262 & 7.8624 & 7.8643 & 7.8345 \\
\hline \multirow{4}{*}{ RMSE } & $\left(\mathrm{F}_{1} \mid \mathbf{M S}_{1}\right)$ & 0.1073 & 0.1273 & 0.1460 & 0.1637 & 0.1728 \\
\hline & $\left(\mathrm{F}_{2} \mid \mathbf{M S}_{2}\right)$ & 0.1002 & 0.1216 & 0.1393 & 0.1544 & 0.1615 \\
\hline & $\left(\mathrm{F}_{3} \mid \mathrm{MS}_{3}\right)$ & 0.1095 & 0.1286 & 0.1436 & 0.1567 & 0.1629 \\
\hline & $(\mathrm{GF} \mid \mathrm{PAN})$ & 0.0802 & 0.0726 & 0.0644 & 0.0536 & 0.0454 \\
\hline \multirow{4}{*}{ STD } & $\left(\mathbf{F}_{1}\right)$ & 0.2346 & 0.2424 & 0.2503 & 0.2546 & 0.2557 \\
\hline & $\left(\mathbf{F}_{2}\right)$ & 0.2394 & 0.2471 & 0.2546 & 0.2579 & 0.2582 \\
\hline & $\left(\mathbf{F}_{3}\right)$ & 0.2466 & 0.2542 & 0.2620 & 0.2655 & 0.2657 \\
\hline & (GF) & 0.2382 & 0.2457 & 0.2535 & 0.2572 & 0.2580 \\
\hline
\end{tabular}


Table 2: statistical indicators of CC, EN, RMSE, STD for fused images resulted through different fusion rules

\begin{tabular}{|c|c|c|c|c|}
\hline & & $\begin{array}{c}\text { Selection of max } \\
\text { abs details }\end{array}$ & $\begin{array}{l}\text { Max average } \\
\text { gradient }\end{array}$ & Replace all MS details \\
\hline \multirow{4}{*}{$\mathrm{CC}$} & $\left(\mathbf{F}_{1} \mid \mathbf{P A N}\right)$ & 0.8963 & 0.9871 & 0.9933 \\
\hline & $\left(\mathbf{F}_{2} \mid \mathbf{P A N}\right)$ & 0.9886 & 0.9894 & 0.9953 \\
\hline & $\left(\mathbf{F}_{3} \mid \mathbf{P A N}\right)$ & 0.9896 & 0.9801 & 0.9957 \\
\hline & (GF|PAN) & 0.9892 & 0.9893 & 0.9951 \\
\hline \multirow{4}{*}{$\mathrm{EN}$} & $\left(\mathbf{F}_{1}\right)$ & 7.8418 & 7.7682 & 0.8227 \\
\hline & $\left(\mathbf{F}_{2}\right)$ & 7.8150 & 7.7689 & 0.7984 \\
\hline & $\left(\mathbf{F}_{3}\right)$ & 7.7128 & 7.6855 & 0.6969 \\
\hline & (GF) & 7.8169 & 7.7649 & 0.8021 \\
\hline \multirow{4}{*}{ RMSE } & $\left(\overline{\left(F_{1} \mid M_{1}\right)}\right.$ & 0.1224 & 0.0950 & 0.1402 \\
\hline & $\left(\mathbf{F}_{2} \mid \mathbf{M S}_{2}\right)$ & 0.1158 & 0.0987 & 0.1336 \\
\hline & $\left(\mathbf{F}_{3} \mid \mathbf{M S}_{3}\right)$ & 0.1225 & 0.0974 & 0.1362 \\
\hline & $(\mathrm{GF} \mid \mathrm{PAN})$ & 0.0849 & 0.0893 & 0.0573 \\
\hline \multirow{4}{*}{ STD } & $\left(\mathbf{F}_{1}\right)$ & 0.2493 & 0.2329 & 0.2427 \\
\hline & $\left(\mathbf{F}_{2}\right)$ & 0.2509 & 0.2308 & 0.2461 \\
\hline & $\left(\mathbf{F}_{3}\right)$ & 0.2538 & 0.2340 & 0.2501 \\
\hline & (GF) & 0.2569 & 0.2383 & 0.2440 \\
\hline
\end{tabular}


Table 3: statistical indicators of CC, EN, RM SE, STD for fused images resulted through different wavelet functions

\begin{tabular}{|c|c|c|c|c|c|c|}
\hline & & Db2 & Db10 & Sym2 & Bior1.1 & Coif2 \\
\hline \multirow{4}{*}{$\mathrm{CC}$} & $\left(\mathrm{F}_{1} \mid \mathrm{PAN}\right)$ & 0.9937 & 0.9932 & 0.9937 & 0.9939 & 0.9933 \\
\hline & $\left(\mathrm{F}_{2} \mid\right.$ PAN $)$ & 0.9958 & 0.9954 & 0.9958 & 0.9959 & 0.9954 \\
\hline & $\left(\mathbf{F}_{3} \mid\right.$ PAN $)$ & 0.9962 & 0.9958 & 0.9962 & 0.9963 & 0.9959 \\
\hline & $(\mathbf{G F} \mid \mathbf{P A N})$ & 0.9941 & 0.9937 & 0.9941 & 0.9941 & 0.9938 \\
\hline \multirow{4}{*}{$\mathrm{EN}$} & $\overline{\left(F_{1}\right)}$ & 7.8307 & 7.8470 & 7.8307 & 7.8003 & 7.8430 \\
\hline & $\left(\mathbf{F}_{2}\right)$ & 7.8515 & 7.8621 & 7.8515 & 7.8184 & 7.8586 \\
\hline & $\left(\mathbf{F}_{3}\right)$ & 7.7167 & 7.7293 & 7.7167 & 7.6755 & 7.7246 \\
\hline & (GF) & 7.8575 & 7.8663 & 7.8575 & 7.8333 & 7.8629 \\
\hline \multirow{4}{*}{ RMSE } & $\left(\mathrm{F}_{\mathbf{1}} \mid \mathbf{M S}_{\mathbf{1}}\right)$ & 0.1494 & 0.1462 & 0.1494 & 0.1504 & 0.1466 \\
\hline & $\left(\mathbf{F}_{2} \mid \mathbf{M S}_{2}\right)$ & 0.1424 & 0.1394 & 0.1424 & 0.1428 & 0.1396 \\
\hline & $\left(\mathbf{F}_{3} \mid \mathbf{M S}_{3}\right)$ & 0.1462 & 0.1436 & 0.1462 & 0.1470 & 0.1438 \\
\hline & $(\mathbf{G F} \mid \mathbf{P A N})$ & 0.0624 & 0.0644 & 0.0624 & 0.0626 & 0.0641 \\
\hline \multirow{4}{*}{ STD } & $\left(\mathbf{F}_{1}\right)$ & 0.2510 & 0.2504 & 0.2510 & 0.2504 & 0.2507 \\
\hline & $\left(F_{2}\right)$ & 0.2550 & 0.2547 & 0.2550 & 0.2547 & 0.2548 \\
\hline & $\left(\mathbf{F}_{\mathbf{3}}\right)$ & 0.2624 & 0.2521 & 0.2524 & 0.2521 & 0.2522 \\
\hline & (GF) & 0.2540 & 0.2534 & 0.2540 & 0.2534 & 0.2536 \\
\hline
\end{tabular}


Table 4: statistical indicators of CC, EN, RMSE, STD for fused images resulted of wavelet-base method, HIS method and brovey transform method.

\begin{tabular}{|c|c|c|c|c|}
\hline & & IHS & Brovey & DWT \\
\hline \multirow{4}{*}{$\mathrm{CC}$} & $\left(F_{1} \mid\right.$ PAN $)$ & 0.9918 & 0.9944 & 0.9931 \\
\hline & $\left(\mathbf{F}_{2} \mid \mathbf{P A N}\right)$ & 0.9963 & 0.9978 & 0.9954 \\
\hline & $\left(\mathrm{F}_{3} \mid \mathbf{P A N}\right)$ & 0.9955 & 0.9958 & 0.9958 \\
\hline & (GF $\mid$ PAN) & 0.9971 & 0.9996 & 0.9937 \\
\hline \multirow{4}{*}{ EN } & $\left(\mathbf{F}_{1}\right)$ & 7.5807 & 7.6716 & 7.8426 \\
\hline & $\left(\mathbf{F}_{2}\right)$ & 7.6263 & 7.6587 & 7.8588 \\
\hline & $\left(\mathbf{F}_{3}\right)$ & 7.4984 & 7.5666 & 7.7252 \\
\hline & (GF) & 7.7141 & 7.6355 & 7.8624 \\
\hline \multirow{4}{*}{ RMSE } & $\left(\mathrm{F}_{1} \mid \mathbf{M S}_{\mathbf{1}}\right)$ & 0.1580 & 0.1607 & 0.1460 \\
\hline & $\left(\mathbf{F}_{2} \mid \mathbf{M S}_{2}\right)$ & 0.1619 & 0.1709 & 0.1393 \\
\hline & $\left(\mathrm{F}_{3} \mid \mathrm{MS}_{3}\right)$ & 0.1613 & 0.1646 & 0.1436 \\
\hline & (GF $\mid$ PAN) & 0.0439 & 0.0165 & 0.0644 \\
\hline \multirow{4}{*}{ STD } & $\left(\mathbf{F}_{1}\right)$ & 0.2487 & 0.2383 & 0.2503 \\
\hline & $\left(\mathbf{F}_{2}\right)$ & 0.2537 & 0.2433 & 0.2546 \\
\hline & $\left(\mathbf{F}_{3}\right)$ & 0.2625 & 0.2534 & 0.2620 \\
\hline & (GF) & 0.2505 & 0.2386 & 0.2533 \\
\hline
\end{tabular}

\section{References}

1. C.Pohl, J.L. Van Genderen "Multisensory image fusion in remote sensing: concepts, methods and applications" int. j. remote sensing, , vol. 19, no. 5, pp823-854,1998

2. María González-Audícana, José Luis Saleta, Raquel García Catalán, and Rafael García "Fusion of Multispectral and Panchromatic Images Using Improved IHS and PCA Mergers Based on Wavelet Decomposition” IEEE Transaction on Geoscience and Remote Sensing, vol. 42, No. 6, June 2004.

3. Myungjin Choi "A New Intensity-Hue-Saturation Fusion Approach to Image Fusion With a Tradeoff Parameter" IEEE Transaction on Geoscience and Remote Sensing, vol.44,No.6, June2006

4. Amolins, K.; Yun Zhang; Dare, $\mathrm{P}$ “Applications of Wavelet Transforms in Image Fusion" Urban Remote Sensing Joint Event 2007 
5. Song Qiang; Wang Jiawen; Zhang Hongbin; "An Overview on Fusion of Panchromatic Image and Multispectral Image" IEEE 2009

6. S. G.Mallat "A theory for Multiresolution Signal Decomposition: The Wavelet Representation" IEEE transaction on pattern analysis and machine intelligence vol.II, No.7. Jully 1989

7. Yao-Hong Tsai; Yen-Han Lee "Wavelet-based image fusion by adaptive decomposition" Eighth International Conference on Intelligent Systems Design and Applications. IEEE, 2008.

8. K.kannan., and S.A Perumal "Optimal Decomposition Level of Discrete Wavelet Transform for Pixel Based Fusion of Multi-focused Images". International Conference on Computational Intelligence and Multimedia Applications 2007

9. J. Honglin , H.TIAN, and Jian LIU'Remote Sensing Image Fusion Based on Average Gradient of wavelet transform" Proceeding of the IEEE International Conference on Mechatronics \& Automation. NiagarFalls, Canada. Jully 2005

10. Li S., Kwok J.T., and Wang Y.," Using the Descrete Wavelet Transform frame transform to merge Landsat TM and SPOT panchromatic images" Information Fusion 3, pp. 17-23 2002

11. Rick S. Blum and Zheng Liu "Multi-Sensor Image Fusion and Its Applications" CRC press Taylor and Francis Group, 2006 